\title{
Inflammatory Cerebrospinal Fluid in Sporadic Creutzfeldt-Jakob Disease
}

\author{
Esther Bui, Eric Ehrensperger, Demetrios J. Sahlas, Brian J. Murray, \\ Catherine Bergeron, Rafael S. Glikstein, Richard Aviv, Hyman M. Schipper
}

\begin{abstract}
Background: Sporadic Creutzfeldt-Jakob disease (CJD) is a fatal, transmissible spongiform encephalopathy characterized by rapidly progressive dementia, myoclonus, ataxia and akinetic mutism. The underlying mechanism is believed to be a conformational change of a native prion protein which characteristically fails to provoke an immune response. Commensurate with the latter, cerebrospinal fluid (CSF) classically exhibits a non-inflammatory profile. Cases: We report two patients with pathologically-proven sporadic CJD presenting with a significant CSF pleocytosis. Conclusion: Although uncommon, the presence of an inflammatory CSF profile should not exclude the diagnosis of sporadic CJD.
\end{abstract}

RÉSUMÉ: Liquide céphalorachidien inflammatoire dans la maladie de Creutzfeldt-Jakob sporadique. Contexte : La maladie de Creutzfeldt-Jakob (MCJ) est une encéphalopathie spongiforme transmissible qui est fatale et qui se caractérise par une démence rapidement progressive, du myoclonus, de l'ataxie et un mutisme akinétique. Le mécanisme sous-jacent est, croit-on, un changement de conformation d'une protéine prion native qui ne provoque donc pas de réponse immunitaire. Le liquide céphalorachidien (LCR) a un profil non inflammatoire chez ces patients. Observations : Nous rapportons les observations faites chez deux patients, dont la MCJ a été prouvée en anatomopathologie, qui présentaient une pleiocytose importante du LCR. Conclusion : Bien que rare, la présence d'un profil inflammatoire du LCR ne devrait pas exclure le diagnostic de MCJ sporadique.

Can. J. Neurol. Sci. 2008; 35:625-629

Transmissible spongiform encephalopathies (TSE) are associated with the accumulation of abnormal protease-resistant proteins (prions) which are isoforms of protease-sensitive normal host cellular proteins. ${ }^{1}$ The protein-only hypothesis, initially promulgated by Prusiner and supplanting an earlier slow-virus etiology, posits a conformational change of the alphahelical component of the normal host cellular protein to a betapleated sheet structure resulting in prion accumulation within, and eventual degeneration of, affected neuronal cells. ${ }^{2}$ Human TSE occurs in both sporadic (non-familial) and, less commonly, inherited forms. Mutant prion proteins have been implicated in Creutzfeldt-Jakob disease (CJD), new variant CJD, GerstmannStraussler-Scheinker disease, familial and sporadic fatal insomnia, and kuru. In these conditions, immune/inflammatory reactions are generally absent in blood, brain parenchyma and cerebrospinal fluid (CSF), perhaps because their pathogeneses do not entail exposure to foreign antigens. In patients with progressive central nervous system (CNS) dysfunction, the presence of a significant CSF pleocytosis generally excludes TSE as a viable diagnostic consideration. In this article, we report two patients with neuropathologically-confirmed sporadic CJD presenting with an inflammatory CSF profile.

\section{Case Reports and Methods}

Patient 1 was a 76-year-old Italian woman who presented to hospital in March 2004 after a fall down a flight of stairs secondary to progressive gait difficulties of six months duration. Her family described a stooped posture with no adventitious movements or cognitive changes. Magnetic resonance imaging (MRI) revealed restricted diffusion over the right parietal cortex (Figure 1). Investigations for stroke risk factors, including

From the Division of Neurology, Department of Medicine (EB, DJS, BJM), Division of Neuroradiology, Department of Medical Imaging (RA), Sunnybrook Health Sciences Centre, and Department of Laboratory Medicine and Pathobiology (CB), University of Toronto, Toronto, Ontario; Departments of Neurology \& Neurosurgery (EE, HMS), and Radiology (RSG), Sir Mortimer B. Davis Jewish General Hospital, McGill University, Montreal, Quebec, Canada.

Received April 7, 2008. Final Revisions Submitted May 22, 2008. Correspondence to: Hyman Schipper, Lady Davis Institute for Medical Research, Sir Mortimer B. Davis Jewish General Hospital, 3755 Cote St. Catherine Road, Montreal, Quebec, H3T 1E2, Canada. 
carotid and transcranial ultrasonography, echocardiogram, fasting lipid profile and coagulation screen, were unremarkable. Her mobility progressively deteriorated and by June 2004 she had become wheelchair-bound. She then rapidly developed myoclonus and alien limb phenomenon of the left arm, shortterm memory impairment, poor concentration, dysarthria and dressing apraxia. A repeat MRI of the brain revealed persistent and progressive restricted diffusion within the right hemisphere (Figure 2). The patient was re-admitted for further investigations. Complete blood count showed no evidence of leukocytosis and renal and liver function tests were normal. Tests for vasculitis, anti-thyroid antibodies, syphilis, and herpes simplex virus were negative. A lumbar puncture performed under fluoroscopy

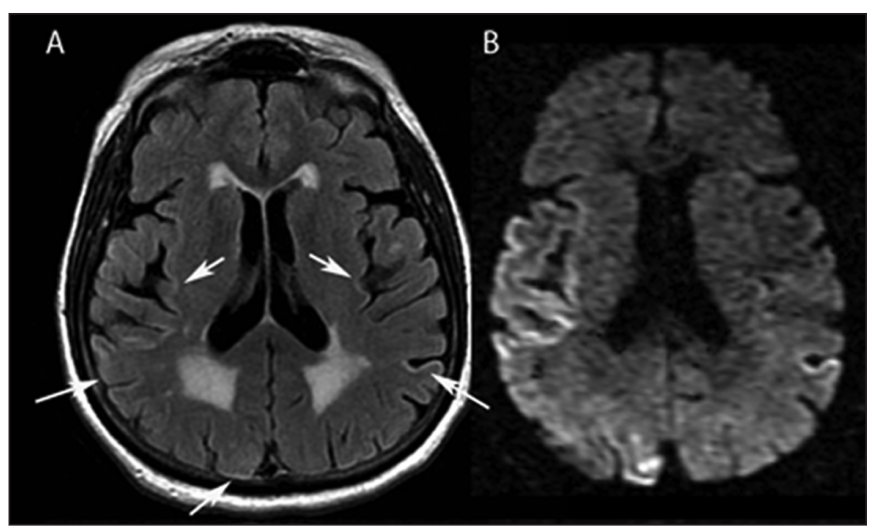

Figure 1: Patient 1. Magnetic resonance imaging reveals (A) subtle cortical hyperintensity involving the bilateral insular region, parietal and right occipital lobes (white arrows) on FLAIR. Confluent nonspecific periventricular and scattered deep white matter T2-weighted signal abnormalities are also evident. B) DWI reveals restricted diffusion within cortical regions appearing hyperintense on FLAIR.

revealed an erythrocyte count of 104 and a white blood cell count of 71 with $90 \%$ neutrophils, $8 \%$ lymphocytes and $2 \%$ monocytes. Cerebrospinal fluid cytology revealed a reactive inflammatory profile. Cerebrospinal fluid protein and glucose concentrations were normal; a 14-3-3 protein determination was not obtained (Table). Bacterial cultures, fungal cultures, mycobacterial and viral studies were negative. An EEG showed diffuse slowing without periodic discharges. A SPECT scan of the brain was normal. A brain biopsy was performed at the right middle frontal gyrus and revealed neuronal loss and astrogliosis. Brain extract treated with proteinase $\mathrm{K}$, separated on $12 \%$ polyacrylamide gel and immunoblotted with anti-PrP antisera revealed a migration pattern of the three major isoforms of proteinase $\mathrm{K}$ resistant PrP type $2^{3}$ (data not shown, but see Figure 4). The neuropathology and Western Blot analysis were diagnostic of CJD. The patient's dementia progressed to akinetic mutism. She died one year after initial presentation. The brain weighed 1190 grams at autopsy. Atheroma in basal vessels was

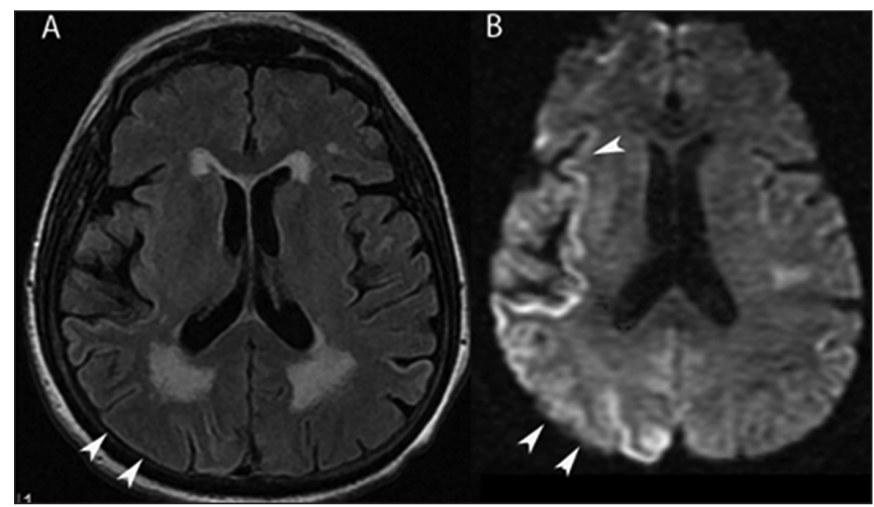

Figure 2: Patient 1. Repeat MRI 5 months after the first study demonstrates persistent and progressive T2 FLAIR (A) and DWI (B) signal abnormalities with more confluent right insular, parietal and occipital involvement (arrowheads).

minimal. There was extensive confluent spongiform change in cortex (Figure 3A), thalamus and striatum. Coarse perivacuolar deposits of PrP immunoreactivity were abundant in these regions (Figure 3B) and in globus pallidus, hippocampus and cerebellar molecular layer. $\operatorname{PrP}($ resistant) immunoreactivity was confirmed by Western blotting (Figure 4). Gliosis was present in the medial thalamus (moderate) and inferior olive (mild). Secondary degenerative changes in the white matter with reactive

Table: Cerebrospinal fluid profiles of CJD Patients 1 and 2

\begin{tabular}{lccc}
\hline & Patient 1 & \multicolumn{2}{c}{ Patient 2 } \\
& LP & $\begin{array}{c}\text { LP\#1 (Day 4 } \\
\text { of admission) }\end{array}$ & $\begin{array}{c}\text { LP\#2 (Day 14 } \\
\text { of admission) }\end{array}$ \\
Appearance & Clear & Clear & Clear \\
Color & Colorless & Colorless & Colorless \\
Protein (g/L) & 0.21 & 0.63 & 0.46 \\
Glucose (mmol/L) & 3.5 & 3.9 & 4.3 \\
Cells & & & \\
-RBCs & 104 & 0 & 0 \\
-WBCs & 71 & 62 & 30 \\
Polymorphonuclear & $90 \%$ & $0 \%$ & $0 \%$ \\
Mononuclear & $10 \%$ & $100 \%$ & $100 \%$ \\
-Lymphocytes & $8 \%$ & $90 \%$ & $85 \%$ \\
-Monocytes & $2 \%$ & $10 \%$ & $15 \%$ \\
Cultures & & & \\
-Bacterial & Negative & Negative & Negative \\
-Viral & Negative & Negative & Negative \\
-Fungal & Negative & Negative & Negative \\
Protein 14-3-3 & Not tested & Not tested & Positive \\
\hline
\end{tabular}




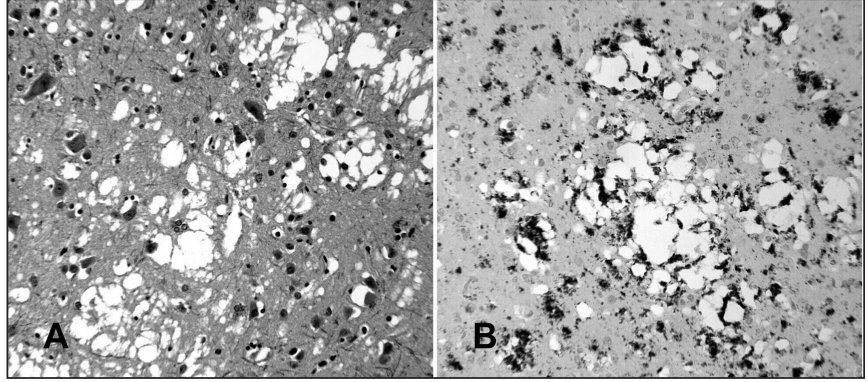

Figure 3: Confluent spongiform change in biopsy specimen from Patient 1 (A). PrP immunodeposits are seen in a coarse perivacuolar pattern (B). $A \& B$, frontal cortex, original magnification x 250. Hematoxylineosin/Luxol fast blue (A) and PrP immunostain (B).

astrocytes, macrophages and axonal swellings were observed at the biopsy site. There was no evidence of an inflammatory or ischemic process.

Patient 2 was a 72-year-old, previously well, Iranian woman who presented with a two-week history of gait instability, apathy and urinary incontinence. Her problem began abruptly with difficulty getting out of a car following a long road trip. Her family sought medical attention for progressive gait impairment. A review of systems revealed an unintentional $15-\mathrm{kg}$ weight-loss over the past year. There was no history of fever or recent illness. She had traveled to Iran three months prior to presentation. The family history was non-contributory for any medical or neurological illnesses. On examination, she appeared apathetic. Her general physical exam was unremarkable. She was alert and oriented. She lacked spontaneous speech but answered questions appropriately. Her language function was normal. Cranial nerve examination was normal. Tone and strength were normal. Deep tendon reflexes were +2 and symmetrical in the upper and lower extremities. Plantar responses were flexor. She had a moderate to severe intention tremor and poor heel-shin testing on the left. Her

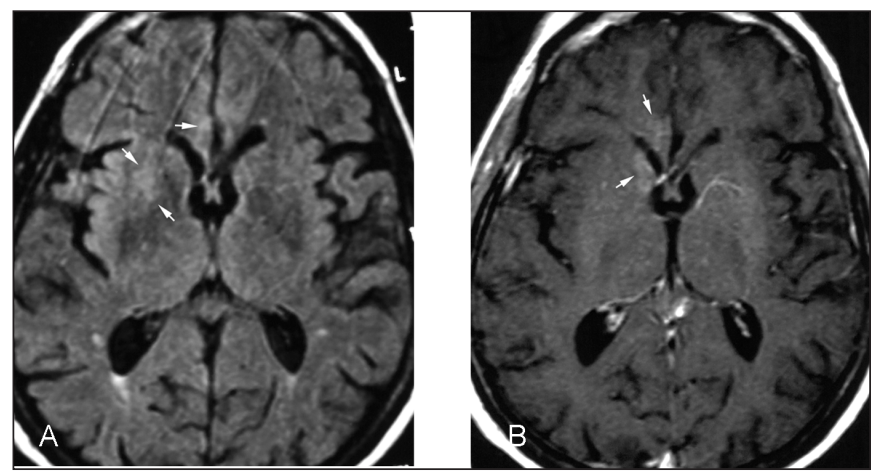

Figure 5: Patient 2. Initial MRI shows hyperintense signal of the right lentiform nucleus on FLAIR (A) and abnormal enhancement of the head of the right caudate nucleus and genu of the corpus callosum on axial T1 with gadolinium $(B)$.

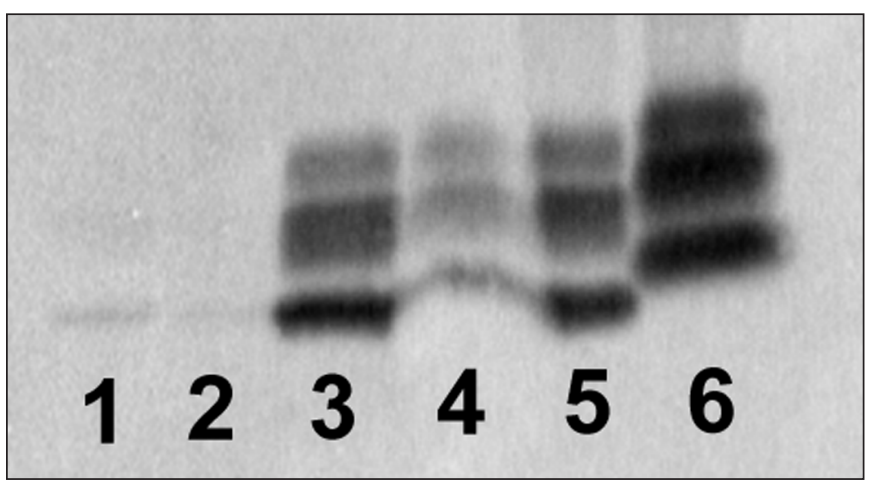

Figure 4: Western Blot for PrP(res) from four autopsied brain regions in Patient 1. A low intensity signal is seen in cingulate (lane 1) and occipital cortex (lane 2). A strong signal is noted in temporal cortex (lane 3 ) and thalamus (lane 4). Lane 5 and 6 depict PrP type 2 banding pattern controls with lowest molecular weight bands at $19 \mathrm{kDa}$ and $21 \mathrm{kDa}$, respectively.

gait was unsteady and required the assistance of one person to prevent falling. Initial routine blood tests were normal. A computed tomogram of the brain showed a $10.8 \mathrm{~mm}$ calcified meningioma adjacent to the right parietal bone without mass effect and mild cerebral atrophy. An MRI scan revealed bilateral small scattered areas of hyperintensity involving the subcortical white matter on T2-weighted imaging. Fluid attenuated inversion recovery (FLAIR) and diffusion weighted imaging (DWI) showed subtle increased signal involving the right head of caudate and rostral corpus callosum. There was contrast enhancement involving the same areas (Figure 5). Within 48 hours of admission, the patient developed dystonic posturing and chorea-like movements of the left arm. On the fourth day of admission, she experienced a sudden deterioration with generalized rigidity and inability to communicate or follow commands. Her vital signs were stable and she remained
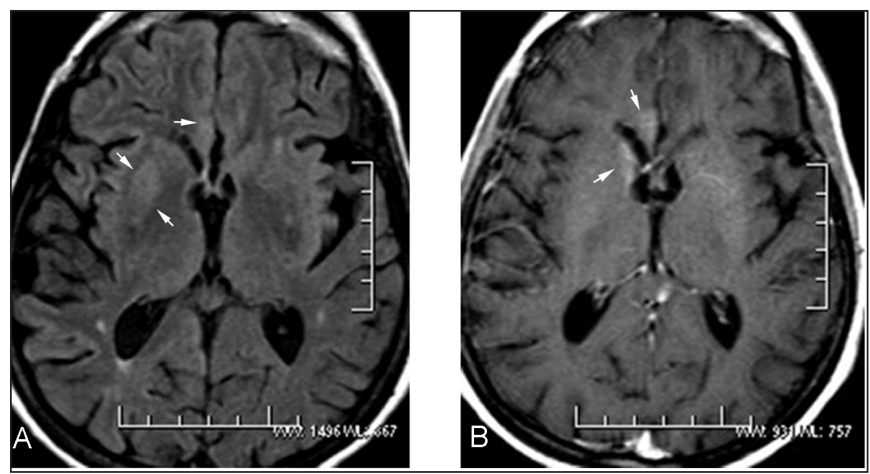

Figure 6: Patient 2. Repeat MRI one month later demonstrates progression of the hyperintense signal of the right lentiform nucleus and corpus callosum on FLAIR (A) and persistent enhancement of the head of the right caudate nucleus and genu of the corpus callosum on axial T1 with gadolinium $(B)$. 


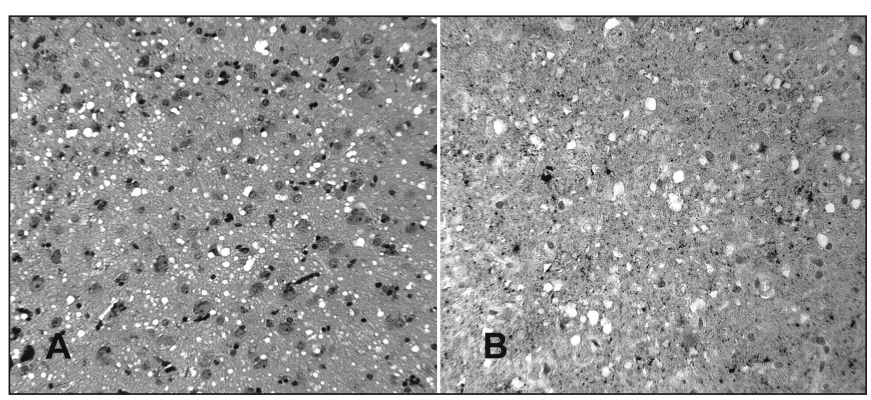

Figure 7: (A) Microvacuolation and mild gliosis in brain biopsy specimen from Patient 2. Hematoxylin-eosin/Luxol fast blue stain. Original magnification $X$ 250. (B) A microvacuolar pattern of PrP(resistant) immunoreactivity is depicted. PrP immunostain. X 400

afebrile. A lumbar puncture performed the same day revealed slightly elevated protein, normal glucose, 62 leucocytes and 0 erythrocytes (Table). The CSF cultures were negative. She was started on acyclovir for possible viral encephalitis. Further blood tests for infectious, metabolic, endocrine, toxic and inflammatory causes were negative. A repeat MRI of the brain demonstrated progression from the previous study. Extensive gyriform hyperintensities were seen involving the right hemisphere and there was increased signal abnormality on FLAIR with persistent gadolinium enhancement of the right head of the caudate nucleus (Figure 6). An EEG showed diffuse symmetrical slow wave activity involving both hemispheres with asymmetrical slow activity seen intermittently over the right temporal region. A repeat spinal tap on day 14 revealed persistent CSF pleocytosis with 30 leukocytes and 0 erythrocytes. Cerebrospinal fluid 14-3-3 protein was positive. An open brain biopsy was performed. The pathology revealed microvacuolation with mild neuronal loss and gliosis. Immunohistochemistry demonstrated a delicate synaptic pattern of $\operatorname{PrP}($ Resistant) deposits throughout the cortex indicative of sporadic CJD (Figure 7). The patient continued to deteriorate and died two months after the onset of her symptoms. An autopsy was not performed.

\section{Discussion}

Sporadic CJD is the most common human transmissible spongiform encephalopathy, occurring at an incidence of one person per million annually. It is a progressive and incurable disease that typically presents with progressive dementia and myoclonus. Visual symptoms, cerebellar findings, pyramidal and extrapyramidal motor features may complete the clinical picture. Akinetic mutism develops during the end stage of the disease with death occurring within one year for the majority of patients. Current diagnostic criteria from the World Health Organization divide sporadic CJD into definite, probable, and possible CJD. ${ }^{4}$ A diagnosis of definite sporadic CJD requires neurohistopathological confirmation of protease-resistant prion protein, in addition to characteristic features of spongiform degeneration, neuronal loss and astrocytic gliosis. ${ }^{5} \mathrm{~A}$ diagnosis of probable sporadic CJD is made by clinical criteria in addition to typical electroencephalogram findings of periodic sharp wave complexes, or the presence of 14-3-3 protein in the CSF. More recently, magnetic resonance imaging has emerged as a promising diagnostic tool. ${ }^{6-8}$

Although the CSF composition is not formally part of the diagnostic criteria, the CSF in CJD is characteristically acellular with normal glucose. ${ }^{9-14}$ Cases with mild pleocytosis (5 to 11 cells per $\mathrm{mm}^{3}$ ) have been infrequently reported. ${ }^{15}$ The CSF protein may be normal or slightly elevated, but rarely greater than $500 \mathrm{mg}$ per deciliter. ${ }^{16}$ Importantly, a cellular CSF suggests alternative diagnoses that may clinically mimic CJD, including tertiary syphilis, subacute sclerosing panencephalitis, Hashimoto's encephalitis, herpes encephalitis, cryptococcal meningitis, and paraneoplastic limbic encephalitis. One study of CJD classified patients under the category of "other disease" based on atypical clinical features and CSF evidence of an inflammatory process. ${ }^{17}$

In the current report, diagnoses of sporadic CJD were confirmed in two patients by classical neuropathology and antiPrP immunochemistry. These individuals underwent open brain biopsy prompted mainly by the 'confounding' inflammatory CSF profiles. In Patient 1, the red blood cell count was elevated in the CSF raising the question of a traumatic tap. However, this possibility can be discounted by the excessive CSF leukocyte:erythrocyte ratio obtained. ${ }^{18}$ Furthermore, in neither case was there any clinical, biochemical, radiological or pathological evidence of a concurrent infectious, inflammatory or ischemic process that could have theoretically provoked a CSF pleocytosis. We hypothesize that a period of accelerated cortical destruction, whether occurring early or late in the course of CJD, may precipitate an inflammatory reaction detectable by fortuitously-timed lumbar puncture. Expanded surface areas of neural damage, as evidenced by enlarging diffusion weighted abnormalities on repeat MRI in both cases, are consistent with this formulation. Both patients exhibited epochs of precipitous clinical deterioration in the absence of stroke risk factors and without evidence of infarction on brain biopsy. Stroke-like progression has previously been described in patients with $\mathrm{CJD}^{19,20}$ and may represent a further (clinical) correlate of accelerated neuronal demise in this condition.

To our knowledge, these are the first two reported cases of an exuberant CSF pleocytosis attributed to definite sporadic CJD. The presence of a significant inflammatory CSF profile, although unusual, does not rule out CJD and may denote a particularly aggressive phase of the illness.

\section{ACKNOWLEDGMENT}

The authors thank Dr. Steffen Albrecht (S.M.B.D. Jewish General Hospital) for assistance with the neuropathological evaluation of Case 2 .

\section{REFERENCES}

1. Oesch B, Westaway D, Walchli M, McKinley MP, Kent SB, Aebersold R, et al. A cellular gene encodes scrapie PrP 27-30 protein. Cell. 1985; 40:735-46.

2. Prusiner SB. Prions. Sci Am. 1984; 251:50-9.

3. Parchi P, Giese A, Capellari S, Brown P, Schulz-Schaeffer W, Windl $\mathrm{O}$, et al. Classification of sporadic Creutzfeldt-Jakob disease based on molecular and phenotypic analysis of 300 subjects. Ann Neurol. 1999; 46:224-33. 
4. Zeidler M. WHO manual for strengthening diagnosis and surveillance of Creutzfeldt-Jakob disease: World Health Organization: 1988.p. 47-51.

5. Kretzschmar HA, Ironside JW, DeArmond SJ, Tateishi J. Diagnostic criteria for sporadic Creutzfeldt-Jakob disease. Arch Neurol. 1996; 53:913-20.

6. Demaerel P, Sciot R, Robberecht W, Dom R, Vandermeulen D, Maes F, et al. Accuracy of diffusion-weighted MR imaging in the diagnosis of sporadic Creutzfeldt-Jakob disease. J Neurol. 2003; 250:222-5.

7. Meissner B, Kortner K, Bartl M, Jastrow U, Mollenhauer B, Schroter A, et al. Sporadic Creutzfeldt-Jakob disease: magnetic resonance imaging and clinical findings. Neurology. 2004; 63:450-6.

8. Shiga Y, Miyazawa K, Sato S, Fukushima R, Shibuya S, Sato Y, et al. Diffusion-weighted MRI abnormalities as an early diagnostic marker for Creutzfeldt-Jakob disease. Neurology. 2004; 63: 443-9.

9. Haywood AM. Transmissible spongiform encephalopathies. N Engl J Med. 1997; 337:1821-8.

10. Johnson RT, Gibbs CJ, Jr. Creutzfeldt-Jakob disease and related transmissible spongiform encephalopathies. N Engl J Med. 1998; 339:1994-2004.

11. Mendez OE, Shang J, Jungreis CA, Kaufer DI. Diffusion-weighted MRI in Creutzfeldt-Jakob disease: a better diagnostic marker than CSF protein 14-3-3? J Neuroimaging. 2003; 13:147-51.

12. Na DL, Suh CK, Choi SH, Moon HS, Seo DW, Kim SE, et al. Diffusion-weighted magnetic resonance imaging in probable Creutzfeldt-Jakob disease: a clinical-anatomic correlation. Arch Neurol. 1999; 56:951-7.
13. Shinobu LA, Frosch MP. Case records of the Massachusetts General Hospital. Case 28-1999. A 68-year-old woman with rapidly progressive dementia and a gait disorder. N Engl J Med. 1999; 341:901-8.

14. Zeidler M, Green A. Advances in diagnosing Creutzfeldt-Jakob disease with MRI and CSF 14-3-3 protein analysis. Neurology. 2004; 63:410-1.

15. Jacobi C, Zerr I, Arlt S, Schröter A, Otto M, Poser S. Cerebral spinal fluid pattern in patients with definite Creutzfeldt-Jakob disease. J Neurol. 2000; 247 Suppl 3:III/14.

16. Goldhammer Y, Bubis JJ, Sarova-Pinhas I, Braham J. Subacute spongiform encephalopathy and its relation to Jakob-Creutzfeldt disease: report on six cases. J Neurol Neurosurg Psychiatry. 1972; 35:1-10.

17. Poser S, Mollenhauer B, Kraubeta A, Zerr I, Steinhoff BJ, Schroeter A, et al. How to improve the clinical diagnosis of CreutzfeldtJakob disease. Brain. 1999; 122 (Pt 12):2345-51.

18. Rowland LP. Merritt's neurology. Philadelphia: Lippincott Williams \& Wilkins; 2000.

19. Obi T, Takatsu M, Kitamoto T, Mizoguchi K, Nishimura Y. [A case of Creutzfeldt-Jakob disease (CJD) started with monoparesis of the left arm]. Rinsho Shinkeigaku. 1996; 36:1245-8.

20. Panagariya A, Jain RS, Sharma AK. Stroke like presentation of Creutzfeldt Jakob disease: an unusual variant. J Assoc Physicians India. 1999; 47:548-50. 